\title{
A New Approach to Active Illumination*
}

\author{
J. P. McDonald \\ R. J. Fryer \\ Machine Perception Research Group \\ University of Strathclyde \\ Glasgow Scotland
}

\author{
J. P. Siebert \\ The Turing Institute \\ Glasgow Scotland
}

\begin{abstract}
The design and development of a versatile active illumination system is described. The necessary system components are related to existing techniques which utilise various forms of structured light to extract range data. The system is composed of a high power stroboscopic illuminator coupled with a high resolution liquid crystal display and a precision actuated mirror. A colour filtering device enables projection colour control. The performance of a prototype system is demonstrated through application to temporal modulation, a range finding technique which relies heavily on the performance of the illuminating system.
\end{abstract}

\section{Introduction}

Machine vision tasks can often be aided by knowledge of depth information. Examples are segmentation, object classification and object recognition. Some tasks, such as robot navigation require depth information as a prerequisite.

Passive techniques for obtaining range data, both monocular (shape from type) and binocular (stereopsis), analyse captured two dimensional image(s) to reconstruct three dimensional details about the observed world. Both suffer from non trivial difficulties.

Active techniques utilise some form of structured light which is projected onto the scene to alleviate many of the problems encountered by passive techniques. Triangulation is normally used to reveal the depth of points in the captured image in a similar way to stereopsis. Without detailing the mathematics involved, it is sufficient to say that given the image coordinates of a point in space, the camera geometry and knowledge of the projected ray to this point, world coordinates can be obtained.

In this paper we describe a projection system suitable for retrieving depth information by a wide range of structured light techniques. Results from the use of the projector and software to implement one of the described techniques demonstrates the system's performance.

\footnotetext{
*This research was carried out as part of the Active Stereo Probe project, IED3/1/2109
} 


\section{Background}

A complete survey of active optical range finding techniques can be found in [1]. One class of technique is active triangulation. Here various types of illumination probe penetrate the environment allowing depth inference from the distortions of the projection.

Aggarwal and Wang [2] present an overview of such active illumination techniques. Techniques are classified by their requirement to form correspondences between projected points and imaged points. This taxonomy is altered and extended here. Techniques without correspondence determination require no disambiguation of the illumination structure.

\subsection{Techniques without correspondence}

The simplest of these techniques is spot ranging. A single projected beam is scanned across the whole scene and a depth measurement made at each point through triangulation. As with any vision system utilising structured light the projected feature(s), in this case a single spot, require(s) identification within the image. This technique can be extended to light striping [3]. Essentially the spot is replaced by a line which is swept across the scene. Constraints can be applied which allow processing of the line in the same manner as for a spot.

A method of obtaining local surface orientation and hence structure can be found in [4]. Here measurement of the orientation change of parallel projected stripes on the image is made, enabling deduction of scene structure through a constraint satisfaction approach.

Structured lighting can also be employed to improve the operation of normally passive algorithms. Enhancement of stereo algorithms through texture projection is reported in [5] and [6]. Although correspondences are made between left and right image elements, no correspondences between projected rays and elements from either image are required.

\subsection{Techniques with correspondence}

Techniques with correspondence require identification of points, lines or grid intersections within the projected pattern, before depth information can be inferred. The correspondence problem of stereo systems can be reposed as the indexing problem of structured light systems. Like solutions to the stereo correspondence problem no technique is guaranteed to work in all situations.

The most basic category uses planar grid projections [7]. Essentially the correspondence problem of stereo recurs in full, with feature points becoming grid intersections. Projective constraints can be applied to reduce ambiguity as can common stereo constraints (epipolar, disparity gradient).

Utilising the projection itself to disambiguate the pattern can alleviate the indexing task somewhat $[8,9]$. If, for example, a grid is projected using horizontal and vertical spaced stripes, one or more markers can be placed within the pattern to unambiguously identify particular points. This spatial labelling approach tends to localise the indexing problem increasing system reliability.

If a striped pattern is employed, it is possible to give each stripe a unique signature in order to alleviate the indexing problem. Each stripe could contain a binary pattern [10], or a colour pattern [11] unique to itself, or at least so 
infrequent enough within the projection as to reduce the probability of disambiguating incorrectly virtually to zero.

The indexing problem can be trivialised using temporal modulation. Here the projected pattern changes over time allowing each projected element to be identified by recording its state during a sequence of projections. The prototype projector is demonstrated using this technique in Section 4.

\section{Projector Design}

This section outlines the design and components of a versatile illumination system applicable to all the range finding techniques described previously. The major system component is a Liquid Crystal Mask which allows complete control of the projected pattern.

\subsection{Liquid Crystal Display Projection}

Recently many companies have introduced a range of LCD video projectors aimed at the executive presenter or home entertainment markets. They tend to vary widely in terms of cost and specification (resolution, projection range and size, dynamic range, colour, update rate, necessary ambient light conditions). It was decided to use such a system (modified as necessary) and a survey was carried out. The most suitable system found was the $\mathrm{GBI}^{1}$ monochrome M2 projector. Originally designed as a presentational aid, the M2 is a flexible basis for an active projection system. The system design is outlined in Figure 1.

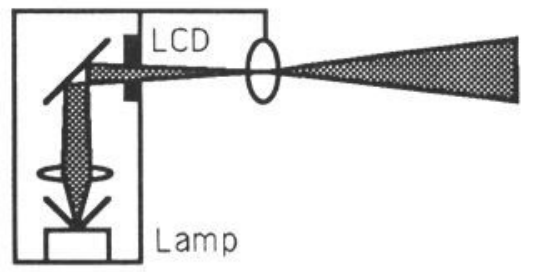

Figure 1: GBI M2 Projector

A $250 \mathrm{~W}$ cold light reflector lamp is directed through a double supertwist chip on glass black/white LCD. The $720 \times 480$ pixel LCD is focussed using an external $160 \mathrm{~mm}$ six element glass lens. Eight grey levels allow a limited dynamic range in projected patterns. However LCD switch times of order $0.5 \mathrm{~s}$ dictate the frequency of any time modulated encoding system with implications for speed of operation, applicability and accuracy. In any closed loop situation where subsequent projections depend on the processing and analysis of current projections the LCD latency is unlikely to hinder overall system response times.

\footnotetext{
${ }^{1}$ GB International Ltd, 82 Alston Drive, Bradwell Abbey, Milton Keynes, United Kingdom, MK13 9HF
} 


\subsection{Design for Universality}

Many of the implementations of the techniques described earlier require controlled background lighting for correct operation. We propose to modify the illumination system to include a stroboscopic lamp which can be coupled to gated cameras synchronised with the strobe flashes. Many methods exist for reducing background effects using such a combination; hence the system should be employable under normal ambient light conditions.

It is also desirable that the system should be capable of interrogating its environment, and not be restricted to one preset direction. Hence our design includes a precision actuated mirror which can be used to direct the projection. Modelling shows that it is feasible, using commercially available actuators, to obtain sub-millimeter accuracy at a projection distance of $5 \mathrm{~m}$.

Further, although the M2 LCD is monochrome we foresee many applications requiring colour control. Hence we have designed a colour filtering system, based on a rotating disk, which can alter the colour of successive projections at video rates. Hence true colour images can be captured in 3 frame times.

With the modifications outlined above our system could be used to implement any of the techniques previously outlined.

\section{Application to Temporal Modulation}

A full explanation of temporal encoding can be found in [12] and [13]. The technique relies on the principle that the deviation of the projected patterns through interaction with the environment, when measured, can reveal scene depth through simple triangulation.

The problem is reduced to one dimension by ensuring projected rows are aligned with camera rows (i.e. the epipolar constraint is applied). Each column in the projected pattern then identifies itself with a unique code through a series of frames. Thus the originating column of any point in the projection, regardless of its position within the scene, can be deduced by analysing the sequence of frames.

The accuracy with which the column can be resolved is defined by the number of frames projected, limited by the resolution of the projector. Therefore coarse depth measurements can be made after only a few projections, and limited processing, while more accurate measurements can be obtained after further projections and subsequent processing.

\subsection{Implementation}

A temporal modulation algorithm has been implemented to demonstrate the flexibility of the prototype projection system. The algorithm extracts the equivalent of disparity from the imaged column number and the deduced LCD column number to allow a depth measurement.

The binary equivalent of the column number of each LCD pixel is used as a basis for the pattern (many other patterns are possible; binary was chosen for ease of implementation). Each pattern and its inverse are sequentially projected. A difference image can then be created which allows stripe detection through thresholding. 


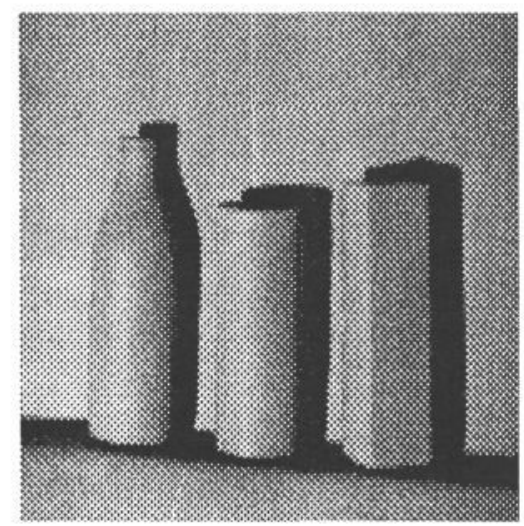

Figure 2: Test Scene

Two constraints are applied during the processing of the sequence. Firstly only approximately the central $75 \%$ of the pattern is imaged in the horizontal direction. This allows detection of scene portions occluded from the projected pattern (their column number would appear as zero which is outside the imaged range), and rejection of other impossible column numbers caused by noise. Secondly resulting disparities are validated using knowledge of possible ranges. Again this eliminates a large proportion of incorrectly generated depths.

\subsection{Results}

The algorithm has been tested on a variety of objects. One example is the scene shown in Figure 2 containing a bottle, a cylinder and a box. An 8 bit representation of the column number was used requiring 16 projections. Figure 3 shows every second bit of the positive version of the sequence. The dense depth map (64x64 measurements) is shown in Figure 4. The map is the direct output of the algorithm described in the previous section, no post-processing was employed. The constraints eliminate noise almost completely. Data for both constraints was supplied manually, although automonous generation would not be difficult. The general shape of the objects have survived well and could easily serve as input to a segmenter or classifier.

\section{Conclusion}

This paper has proposed a flexible illumination system allowing structured lighting suitable for a variety of range finding techniques. The main system components are the LC mask, stoboscopic illuminator and the actuated mirror.

The system has been successfully demonstrated through its application to temporal modulation, which, in terms of illumination system performance, is one of the most demanding applications possible. Firstly, the projection has to vary with time. Previous systems using multiple slides required slide registration. Here registration of successive projections is inherent. Secondly, the 

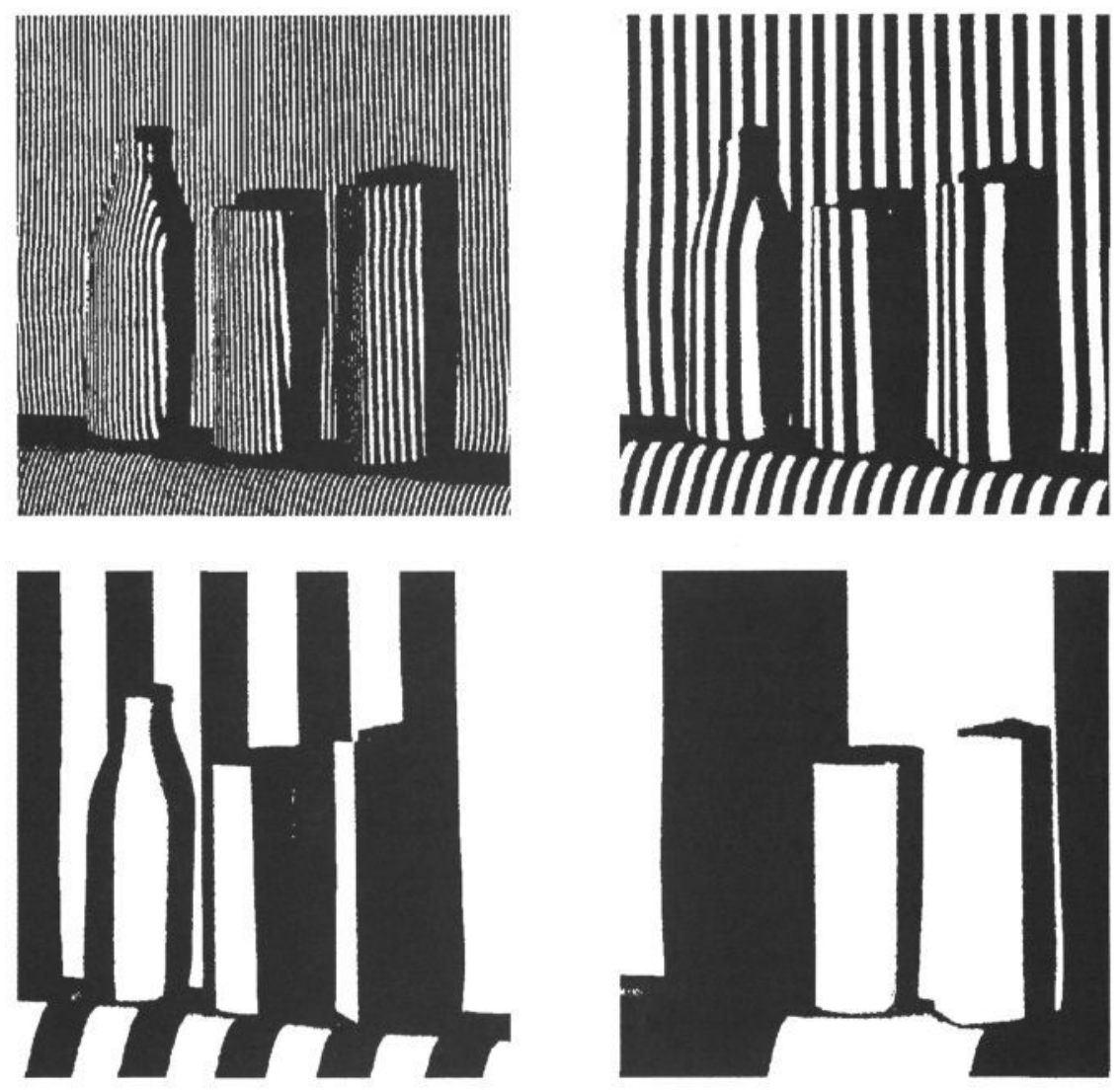

Figure 3: Stripes extracted from Bits 0,2,4 and 6

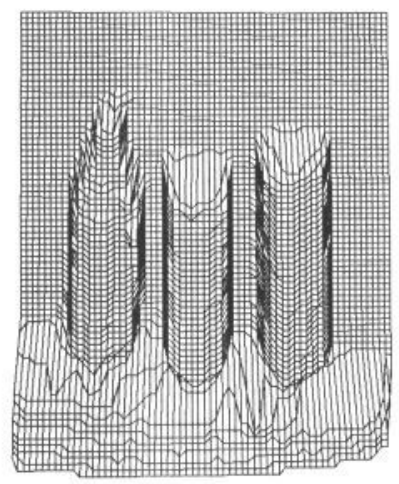

Figure 4: Range Map 
contrast ratio of the projection has to be high enough to enable stripe extraction at the highest spatial frequency. Bit 0 in Figure 3 shows that this has been achieved by the prototype system.

Development of the system and evaluation of its performance will continue, investigating for example the loss of spatial resolution through defocussing of the projection. The use of the system in dynamic scenes is also possible and will be a future topic of research.

\section{References}

[1] P. J. Besl. Active, optical range imaging sensors. In Machine Vision and Applications, 1988.

[2] J. K. Aggarwal and Y. F. Wang. Inference of object surface structure from structured lighting - an overview. In Machine Vision. Academic Press, 1988.

[3] G. J. Agin and T. O. Binford. Computer description of curved objects. In International Joint Conference on Artificial Intelligence, 1973.

[4] A. Mitiche Y. F. Wang and J. K. Aggarwal. Inferring local surface orientation with the aid of grid coding. In Workshop on Computer Vision: Representation and Control, 1985.

[5] J. P. Siebert and C. W. Urquhart. Active stereo : texture enhanced reconstruction. IEE Electronics Letters, 26(7), 1990.

[6] K. H. Cheung and M. D. Fox. Three-dimensional reconstruction of the craniofacial surface with stereopair images. In CH IEEE, 1989.

[7] G. Stockman and G. Hu. Sensing 3D surface patches using a projected grid. In Proceedings of Computer Vision and Pattern Recognition, 1986.

[8] J. Le Moigne and A. M. Waxman. Projected light grids for short range navigation of autonomous robots. In Pattern Recognition, 1984.

[9] R. L. Keizer and S. M. Dunn. Marked grid labelling. In IEEE Proceedings of Computer Vision and Pattern Recognition, 1989.

[10] P. Vuylsteke and A. Oosterlinck. Range image aquisition with a simgle binary-encoded light pattern. IEEE Transactions on Pattern Analysis and Machine Intelligence, 12(2), 1990.

[11] K. L. Boyer and A. C. Kak. Colour-encoded structured light for rapid active ranging. IEEE Transactions on Pattern Analysis and Machine Intelligence, 9(1), 1987.

[12] K. Sato and S. Inokuchi. Range-imaging system utilising nematic liquid crystal mask. In 1st International Conference on Computer Vision, 1987.

[13] J. L. Posdamer and M. D. Altschuler. Surface measurement by spaceencoded projected beam systems. Computer Graphics and Image Processing, 18, 1982. 